\title{
Low Energy Availability in Athletes 2020: An Updated Narrative Review of Prevalence, Risk, Within-Day Energy Balance, Knowledge, and Impact on Sports Performance
}

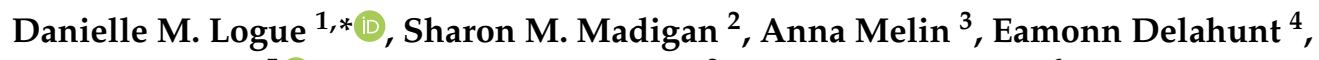 \\ Mirjam Heinen ${ }^{5}\left(\mathbb{D}\right.$, Sarah-Jane Mc Donnell ${ }^{2}$ and Clare A. Corish ${ }^{6}$ \\ 1 School of Public Health, Physiotherapy and Sports Science, University College Dublin, Belfield, \\ V04 V1W8 Dublin 4, Ireland \\ 2 Sport Ireland Institute, Sports Campus Ireland, Abbotstown, D15 PNON Dublin, Ireland; \\ smadigan@instituteofsport.ie (S.M.M.); sjmcdonnell@instituteofsport.ie (S.-J.M.D.) \\ 3 Department of Sports Science, Linnaeus University, 39234 Kalmar, Sweden; anna.melin@lnu.se \\ 4 School of Public Health, Physiotherapy and Sports Science and Institute for Sport and Health, University \\ College Dublin, V04 V1W8 Dublin, Ireland; eamonn.delahunt@ucd.ie \\ 5 Assistant Professor Mirjam Heinen, School of Public Health, Physiotherapy and Sports Science, University \\ College Dublin, V04 V1W8 Dublin, Ireland; mirjam.heinen@ucd.ie \\ 6 Associate Professor Clare Corish, School of Public Health, Physiotherapy and Sports Science, University \\ College Dublin, V04 V1W8 Dublin, Ireland; clare.corish@ucd.ie \\ * Correspondence: danielle.logue@ucdconnect.ie; Tel.: 00-353-86-730-6607
}

Received: 27 February 2020; Accepted: 18 March 2020; Published: 20 March 2020

\begin{abstract}
Low energy availability (EA) underpins the female and male athlete triad and relative energy deficiency in sport (RED-S). The condition arises when insufficient calories are consumed to support exercise energy expenditure, resulting in compromised physiological processes, such as menstrual irregularities in active females. The health concerns associated with longstanding low EA include menstrual/libido, gastrointestinal and cardiovascular dysfunction and compromised bone health, all of which can contribute to impaired sporting performance. This narrative review provides an update of our previous review on the prevalence and risk of low EA, within-day energy deficiency, and the potential impact of low EA on performance. The methods to assess EA remain a challenge and contribute to the methodological difficulties in identifying "true" low EA. Screening female athletic groups using a validated screening tool such as the Low Energy Availability in Females Questionnaire (LEAF-Q) has shown promise in identifying endurance athletes at risk of low EA. Knowledge of RED-S and its potential implications for performance is low among coaches and athletes alike. Development of sport and gender-specific screening tools to identify adolescent and senior athletes in different sports at risk of RED-S is warranted. Education initiatives are required to raise awareness among coaches and athletes of the importance of appropriate dietary strategies to ensure that sufficient calories are consumed to support training.
\end{abstract}

Keywords: low energy availability; relative energy deficiency in sport; health and performance

\section{Introduction}

Considerable research has been undertaken to understand the health and performance consequences of relative energy deficiency in sports (RED-S), a condition frequently observed among high performing male and female athletes $[1,2]$. It is widely acknowledged that low energy availability (EA), described as inadequate energy intake relative to exercise energy expenditure, is the main factor 
triggering the unfavourable health and performance consequences associated with RED-S [3]. Since the publication of the International Olympic Committee (IOC) consensus papers on RED-S in 2014 and 2018 [1,2], scientific evidence for the risk of and performance consequences of low EA has grown [4,5].

Although an understanding of the physiological effects of low EA in male athletes remains limited, a recent cross-sectional study conducted on males participating in endurance training reported that regular exposure to high levels of chronic intense and long duration training is associated with a decreased libido score. [6]. Therefore, the IOC and other researchers in the field [7] advise that research should now focus on the energy demands and performance criteria of males engaged in a range of sports as the majority of research in the past has focused on women [2].

Following publication of our 2018 review on the prevalence of low EA and associated health and performance consequences [7], the volume of research on RED-S has substantially increased (peer-reviewed articles in $2018(n=21), 2019(n=24)$ vs. $2016(n=6)$ and $2017(n=10))$. The purpose of this investigation is to identify other potential methods for assessing low EA given that the current methods used remain challenging. Furthermore, the review highlights recent data on the prevalence and risk of low EA, within-day energy deficiency and the health and sporting performance consequences associated with low EA. Furthermore, the published literature on awareness and knowledge of RED-S among athletes and coaches has been evaluated to identify gaps in the practical application of low EA education in sports [8-11].

\section{Methodology}

This is an update of a narrative review [7] which was conducted using targeted database searches, for example, PubMed, Google Scholar and Web of Science. Combinations of the following key search terms were included: athlete, EA, low EA, low EA risk, within-day energy balance, low EA knowledge and awareness of low EA, nutrition education/diet intervention and RED-S. Articles published between 2017 and 2019 were considered if they were published in English, available in full text and were conducted among trained or exercising human subjects. The inclusion criteria was as follows: only studies that quantified EA by assessing energy intake, exercise energy expenditure (EEE) and body composition and that investigated symptoms associated with low EA within the text of the manuscript were included in this review. The reference lists of retrieved articles were also reviewed to identify any articles not identified by the database searches. Animal studies were excluded. The quality and strength of the supporting evidence were graded according to the criteria of the Scottish Intercollegiate Guidelines Network (SIGN) [12]. The SIGN grading criteria include the assessment of study design and its ability to minimise the possibility of bias as well as an evaluation of the methodological quality, quantity, consistency, and applicability of a study's results to the evidence base.

\section{Results}

\subsection{Low Energy Availability}

Recent studies have investigated the prevalence of low EA in various sports [8,13-22]. Prevalence ranges from $22 \%$ to $58 \%$ (Table 1). It is apparent that low EA exists in males as well as females as evidenced by studies conducted in male road cyclists [9] and elite distance athletes [23-25]. Nonetheless, accurate estimation of the prevalence of low EA remains problematic due to continuing variability in the methods used to estimate EA (Table 2). Furthermore, there are some limitations to the application of clinically low EA $<30 \mathrm{kcal} / \mathrm{kg}$ fat free mass (FFM)/kg/day [3] in cross-sectional studies. Self-reported nutritional data in free-living athletes have failed to find clear thresholds or associations between EA and objective measures of energy conservation or health impairment such as disruption to metabolic hormones [24,26] and menstrual disturbances [27-29]. Most recently (Table 3), athletes are being screened for symptoms of low EA using questionnaires that screen for physiological symptoms associated with the Female Athlete Triad (Triad) and RED-S, an example of which is the Low EA in Females Questionnaire (LEAF-Q) [30]. The current evidence implies that clinical, individual assessment 
of the diagnostic criteria of the Triad [31], the LEAF-Q [30] and the RED-S clinical assessment tool (RED-S CAT) [32] is necessary. This assessment should be conducted with an evaluation of disordered eating behaviours and reproductive biomarkers (blood biochemical assessment of sex-hormones and prospective collection of oestrogen and progesterone during one or more menstrual cycles). This may represent a more objective and accurate indicator of optimal EA for health in females than the estimation of EA using dietary and training logs [24]. Nevertheless, further research is warranted as there is limited research on the effect of hormonal contraception on physiological function and biochemical assessment of low EA/RED-S. 
Table 1. Estimated prevalence of low energy availability in various sport groups (Jan 2017-May 2019).

\begin{tabular}{|c|c|c|c|c|c|c|c|c|c|}
\hline Obser & $\begin{array}{l}\text { Author } \\
\text { tional Studies }\end{array}$ & Sex & & Size & Athletes & $\begin{array}{l}\text { Mean Age } \\
\text { (y) }\end{array}$ & $\begin{array}{c}\text { Mean } \pm \text { SD EA } \\
(\text { kcal/kg FFM/Day)* }\end{array}$ & $\begin{array}{l}\text { Subjects with } \\
\text { Low EA* }(\%)\end{array}$ & Comments \\
\hline 2019 & $\begin{array}{l}\text { McCormack et } \\
\text { al. [25] }\end{array}$ & $\mathrm{M} / \mathrm{F}$ & $\begin{array}{l}107 \\
27 \\
33 \\
23 \\
24\end{array}$ & $\begin{array}{c}\text { M } \\
\text { F } \\
\text { M Ctrl } \\
\text { F Ctrl }\end{array}$ & $\begin{array}{l}\text { Cross-country } \\
\text { skiers and } \\
\text { Ctrl group }\end{array}$ & 18 & $\begin{array}{c}\text { M:36 } \pm 16 \\
\text { F: } 37 \pm 21 \\
\text { M Ctrl: } 42 \pm 15 \\
\text { F Ctrl: } 40 \pm 21\end{array}$ & N/A & $\begin{array}{c}44 \% \text { had EA } 30-45 \mathrm{kcal} / \mathrm{kg} \text { FFM } / \text { day } \\
22 \% \text { had EA }<30 \mathrm{kcal} / \mathrm{kg} \mathrm{FFM} / \text { day. } \\
40 \% \text { MD and } 65 \% \text { at risk of LEA. } \\
\text { No associations between MD and EA }(p=0.17) \\
\text { or LEAF-Q score and EA }(p=0.11) \text {. } \\
\text { F athletes whole-body BMD higher vs. F } \\
\text { Ctrl. Higher dietary restraint score in } \\
\text { athletes vs. Ctrl. } \\
\text { Higher eating concern score in M athletes vs. M } \\
\text { Ctrl. Higher shape concern score in F athletes } \\
\text { vs. M athletes. }\end{array}$ \\
\hline 2019 & $\begin{array}{c}\text { Zabriskie et } \\
\text { al. [19] }\end{array}$ & $\mathrm{F}$ & 20 & & $\begin{array}{l}\text { NCAA } \\
\text { Division II } \\
\text { lacrosse } \\
\text { athletes }\end{array}$ & 20 & $\begin{array}{l}\text { Off season I: } 30 \pm 11 \\
\text { Off season II: } 26 \pm 11 \\
\text { Pre-season: } 23 \pm 9 \\
\text { In season I: } 29 \pm 10 \\
\text { In season II: } 29 \pm 9\end{array}$ & $\mathrm{~N} / \mathrm{A}$ & $\begin{array}{l}\text { Post hoc comparisons showed that 'Pre- } \\
\text { season' trended toward a lower EA than in } \\
\text { 'off season I' ( } p=0.058) \text { and 'in season II' } \\
\qquad(p=0.057) .\end{array}$ \\
\hline 2018 & Braun et al. & F & 56 & & $\begin{array}{l}\text { Elite } \\
\text { soccer } \\
\text { players }\end{array}$ & 15 & N/A & 53 & $\begin{array}{l}\text { Caloric deficit, low carbohydrate and fluid } \\
\text { intakes were observed. }\end{array}$ \\
\hline 2018 & $\begin{array}{l}\text { Cherian et } \\
\text { al. [20] }\end{array}$ & $\mathrm{M} / \mathrm{F}$ & 21 & M & $\begin{array}{l}\text { Junior } \\
\text { national- } \\
\text { level } \\
\text { soccer } \\
\text { players }\end{array}$ & 12 & N/A & $\begin{array}{l}\text { M: } 24 \\
\text { F: } 58\end{array}$ & $\begin{array}{l}4 \text { of } 5 \mathrm{M} \text { and } 7 \text { of } 11 \mathrm{~F} \text { with low EA were } \\
\qquad 16 \text { years of age. }\end{array}$ \\
\hline 2018 & $\begin{array}{c}\text { Costa et al. } \\
\text { [16] }\end{array}$ & $\mathrm{F}$ & $\begin{array}{l}19 \\
21\end{array}$ & $\mathrm{~F}$ & $\begin{array}{l}\text { Collegiate } \\
\text { synchronized } \\
\text { swimmers }\end{array}$ & 20 & $26 \pm 13$ to $30 \pm 13$ & N/A & $\begin{array}{c}\text { Estimated EA was associated with } \\
\text { measured RMR. } \\
\text { No association between } \\
\text { EA and RMR ratio independent of the } \\
\text { prediction equation } \text { used }^{* *}\end{array}$ \\
\hline 2018 & $\begin{array}{l}\text { Heikura et } \\
\text { al. [23] }\end{array}$ & $\mathrm{M} / \mathrm{F}$ & $\begin{array}{l}48 \\
21 \\
27\end{array}$ & $\begin{array}{c}M \\
F\end{array}$ & $\begin{array}{l}\text { Elite } \\
\text { distance } \\
\text { athletes }\end{array}$ & $\begin{array}{l}\text { M: } 27 \\
\text { F: } 26\end{array}$ & $\begin{array}{l}\mathrm{M}: 36 \pm 6 \\
\mathrm{~F}: 33 \pm 7\end{array}$ & N/A & $\begin{array}{l}\text { No associations between } \mathrm{EA} \text { and the } \\
\text { magnitude of relative change in serum } \mathrm{Hb} \text { mass. }\end{array}$ \\
\hline
\end{tabular}




\begin{tabular}{|c|c|c|c|c|c|c|c|c|c|}
\hline \multirow{2}{*}{\multicolumn{2}{|c|}{$\begin{array}{c}\text { Year Author } \\
\text { Observational Studies }\end{array}$}} & \multirow[t]{2}{*}{ Sex } & \multicolumn{2}{|c|}{ Sample Size } & \multirow[t]{2}{*}{ Athletes } & \multirow[t]{2}{*}{ Mean Age (y) } & \multirow{2}{*}{$\begin{array}{c}\text { Mean } \pm \text { SD EA } \\
\text { (kcal/kg FFM/Day)* }\end{array}$} & \multirow{2}{*}{$\begin{array}{l}\text { Subjects with } \\
\text { Low EA* }(\%)\end{array}$} & \multirow[t]{2}{*}{ Comments } \\
\hline & & & & & & & & & \\
\hline \multirow[t]{4}{*}{2018} & Heikura et & $\mathrm{M} / \mathrm{F}$ & 59 & & Elite distance & M: 27 & N/A & M: 25 & Lower oestradiol, total testosterone, $\mathrm{T} 3$ and \\
\hline & al. [24] & & 24 & M & athletes & F: 26 & & F: 31 & BMD in MD (37\%) and low testosterone \\
\hline & & & 35 & $\mathrm{~F}$ & & & & & $(40 \%)$ athletes. \\
\hline & & & & & & & & & $\begin{array}{l}\text { Bone injuries: } \sim 4.5 \text { times more prevalent } \\
\text { in } \mathrm{MD} \text { and low testosterone athletes. }\end{array}$ \\
\hline \multirow{4}{*}{2018} & & & & & Children and & & & & \\
\hline & & & & & adolesc. & & CM:54 \pm 9 & & Lower EA in $\mathrm{M}$ and $\mathrm{F}$ athletes vs. $\mathrm{M}$ and $\mathrm{F}$ \\
\hline & Silva et al. & $\mathrm{M} / \mathrm{F}$ & $\begin{array}{l}82 \\
21\end{array}$ & 61 & acrobatic & M/F children: 11 & CF: $46 \pm 9$ & N/A & Ctrl. \\
\hline & [13] & & $\begin{array}{l}21 \\
M\end{array}$ & $\mathrm{~F}$ & & & $\begin{array}{c}\mathrm{AM}: 45 \pm 15 \\
\mathrm{AF}: 33 \pm 9\end{array}$ & & $\begin{array}{l}\text { Most participants did not eat or drink } \\
\text { during or immediately after training. }\end{array}$ \\
\hline 2018 & $\begin{array}{c}\text { Zanders et } \\
\text { al. [17] }\end{array}$ & $\mathrm{F}$ & 13 & & $\begin{array}{l}\text { Collegiate } \\
\text { basketball } \\
\text { players }\end{array}$ & 20 & 0 & $\mathrm{~N} / \mathrm{A}$ & EA did not change across the season. \\
\hline 2017 & $\begin{array}{l}\text { Brown et al. } \\
\quad[15]\end{array}$ & $\mathrm{F}$ & 25 & & $\begin{array}{l}\text { Pre-professional } \\
\text { contemporary } \\
\quad \text { dancers }\end{array}$ & 21 & $\begin{array}{c}\text { 7-day EA: } 26 \pm 13 \\
\text { Week EA: } 24 \pm 10 \\
\text { Weekend EA: } 36 \pm 21\end{array}$ & N/A & \\
\hline 2017 & $\begin{array}{l}\text { Ong et al. } \\
\text { [18] }\end{array}$ & $\mathrm{F}$ & 9 & & $\begin{array}{l}\text { Dragon boat } \\
\text { athletes }\end{array}$ & 23 & $23.7 \pm 13$ & N/A & $\begin{array}{l}\text { Eight of } 9 \text { subjects had EA }<45 \mathrm{kcal} / \mathrm{kg} \\
\text { FFM/day, with } 6<30 \mathrm{kcal} / \mathrm{kg} \mathrm{FFM/day.}\end{array}$ \\
\hline \multirow[t]{5}{*}{2017} & Silva et al. & M & 151 & & Rink-hockey ${ }^{* * *}$ & Children: 10 & Children: $48 \pm 89$ & N/A & Lower EI and higher EEE in athletes vs. \\
\hline & & & 38 & Children & players and & Adolesc.: 14 & Adolesc.: $50 \pm 11$ & & Ctrl; resulting in some cases of LEA in \\
\hline & & & 34 & Adolesc. & group & & Children Ctrl: $54 \pm 9$ & & Athletes. \\
\hline & & & 43 & $\begin{array}{l}\text { Children } \\
\text { Ctrl }\end{array}$ & & & Adolesc. Ctrl: $55 \pm 18$ & & \\
\hline & & & 36 & $\begin{array}{l}\text { Adolesc. } \\
\text { Ctrl }\end{array}$ & & & & & \\
\hline $\begin{array}{l}\text { Abbrev } \\
\text { Hb; ha } \\
\text { athletic } \\
\text { Harris- }\end{array}$ & $\begin{array}{l}\text { Adolesc: } \\
\text { bin; EA: } \\
\text { ation; RMI } \\
\text { ict equatio } \\
\text { t tissue co }\end{array}$ & ent & bon & ral der & $\begin{array}{l}\text {; Ctrl: control; } \mathrm{F} \\
\text { vailability in fer } \\
\text { viation * <30 kc } \\
\text { asured and pred }\end{array}$ & A: energy avai & $\begin{array}{l}\text { ty; EEE: exercise ene } \\
\text { M: male; MD; mens } \\
\text { diction equations in } \\
\text { Inningham equation }\end{array}$ & $\begin{array}{l}\text { expenditure; EI } \\
\text { l dysfunction; I } \\
\text { led the ratio bet } \\
\text { RMR ratio), the }\end{array}$ & $\begin{array}{l}\text { ergy intake; F: female; FFM: fat-free mass; } \\
\text { : not available; NCAA: national collegiate } \\
n \text { measured and predicted RMR using the } \\
\text { io between measured and predicted RMR }\end{array}$ \\
\hline
\end{tabular}


Table 2. Methods used to assess energy intake and exercise energy expenditure as part of an assessment of energy availability, disordered eating, reproductive function, bone mineral density, body composition and biochemical variables (Jan 2017-May 2019).

\begin{tabular}{|c|c|c|c|c|c|c|c|c|c|c|}
\hline Year & Sectional and & $\begin{array}{c}\text { Participants } \\
\text { (n) }\end{array}$ & $\begin{array}{l}\text { Energy } \\
\text { Intake }\end{array}$ & $\begin{array}{c}\text { Exercise } \\
\text { Energy } \\
\text { Expenditure }\end{array}$ & $\begin{array}{l}\text { DE } \\
\text { Met }\end{array}$ & $\begin{array}{l}\text { Reproductive } \\
\text { Health } \\
\text { s Used }\end{array}$ & BMD & $\begin{array}{c}\text { Body } \\
\text { Composition }\end{array}$ & $\begin{array}{c}\text { Biochemical } \\
\text { Parameters } \\
\text { Assessed }\end{array}$ & $\begin{array}{c}\text { Other } \\
\text { Parameters } \\
\text { Assessed }\end{array}$ \\
\hline 2019 & $\begin{array}{c}\text { Civil et al. } \\
\text { [14] }\end{array}$ & $\begin{array}{l}20 \text { ballet } \\
\text { dancers }\end{array}$ & $\begin{array}{l}\text { Prospective } \\
\text { weighed } \\
\text { dietary } \\
\text { record }\end{array}$ & Accelometer & TFE-Q & $\begin{array}{l}\text { Menstrual } \\
\text { history } \\
\text { questionnaire } \\
\text { and LEAF-Q }\end{array}$ & DXA & DXA & Vitamin D & $\begin{array}{l}\text { Healthier } \\
\text { dance } \\
\text { practice } \\
\text { national } \\
\text { survey }\end{array}$ \\
\hline 2019 & $\begin{array}{c}\text { McCormack } \\
\text { et al. [25] }\end{array}$ & $\begin{array}{c}107 \\
27 \mathrm{M} \\
\text { runners } \\
33 \mathrm{~F} \text { runners } \\
23 \mathrm{M} \\
\text { controls } \\
24 \mathrm{~F} \text { controls }\end{array}$ & FFQ & Activity $\log$ & EDE-Q & $\mathrm{N} / \mathrm{A}$ & DXA & DXA & $\mathrm{N} / \mathrm{A}$ & N/A \\
\hline 2018 & $\begin{array}{c}\text { Black et al. } \\
\text { [33] }\end{array}$ & $\begin{array}{c}38 \\
\text { recreational } \\
\text { athletes }\end{array}$ & $\begin{array}{c}\text { Prospective } \\
\text { weighed } \\
\text { dietary } \\
\text { record }\end{array}$ & Activity $\log$ & N/A & $\begin{array}{l}\text { Menstrual } \\
\text { function } \\
\text { questions in } \\
\text { the LEAF-Q }\end{array}$ & $\mathrm{N} / \mathrm{A}$ & $\begin{array}{c}\text { Bio- } \\
\text { impedance }\end{array}$ & $\begin{array}{c}\text { Serum } \\
\text { cholesterols, } \\
\text { cortisol, } \\
\text { progesterone } \\
\text { and T3. } \\
\text { Salivary } \\
\text { testosterone }\end{array}$ & N/A \\
\hline 2018 & $\begin{array}{c}\text { Braun et al. } \\
\text { [21] }\end{array}$ & $\begin{array}{c}56 \text { F soccer } \\
\text { players }\end{array}$ & $\begin{array}{l}\text { Prospective } \\
\text { weighed } \\
\text { dietary } \\
\text { record }\end{array}$ & Activity log & N/A & N/A & $\mathrm{N} / \mathrm{A}$ & $\begin{array}{c}\text { Bio- } \\
\text { impedance }\end{array}$ & $\begin{array}{c}\text { Serum iron } \\
\text { and } \\
\text { ferritin }\end{array}$ & N/A \\
\hline 2018 & $\begin{array}{c}\text { Cherian et } \\
\text { al. [20] }\end{array}$ & $\begin{array}{c}40 \text { soccer } \\
\text { players } \\
21 \mathrm{M} \\
19 \mathrm{~F}\end{array}$ & $\begin{array}{c}\text { Prospectivew } \\
\text { dietary } \\
\text { record }\end{array}$ & $\begin{array}{l}\text { ighed } \\
\text { HR monitors }\end{array}$ & N/A & N/A & $\mathrm{N} / \mathrm{A}$ & $\begin{array}{c}\text { 4-site } \\
\text { skinfold } \\
\text { measurements }\end{array}$ & N/A & N/A \\
\hline
\end{tabular}




\begin{tabular}{|c|c|c|c|c|c|c|c|c|c|c|}
\hline Year & Sectional and & $\begin{array}{c}\text { Participants } \\
\text { (n) }\end{array}$ & $\begin{array}{l}\text { Energy } \\
\text { Intake }\end{array}$ & $\begin{array}{c}\text { Exercise } \\
\text { Energy } \\
\text { Expenditure }\end{array}$ & $\mathbf{M}$ & $\begin{array}{l}\text { Reproductive } \\
\text { Health } \\
\text { Is Used }\end{array}$ & BMD & $\begin{array}{c}\text { Body } \\
\text { Composition }\end{array}$ & $\begin{array}{c}\text { Biochemical } \\
\text { Parameters } \\
\text { Assessed }\end{array}$ & $\begin{array}{c}\text { Other } \\
\text { Parameters } \\
\text { Assessed }\end{array}$ \\
\hline 2018 & $\begin{array}{c}\text { Costa et al. } \\
\text { [16] }\end{array}$ & $\begin{array}{c}21 \mathrm{~F} \\
\text { collegiate } \\
\text { synchronized } \\
\text { swimmers }\end{array}$ & $\begin{array}{c}\text { Prospective } \\
\text { dietary } \\
\text { record }\end{array}$ & Activity log & N/A & N/A & DXA & $\begin{array}{l}\text { 4- and 7-site } \\
\text { skinfold } \\
\text { measurements } \\
\text { and DXA }\end{array}$ & N/A & $\begin{array}{l}\text { RMR using } \\
\text { indirect } \\
\text { calorimetry }\end{array}$ \\
\hline 2019 & $\begin{array}{c}\text { Zabriskie et } \\
\text { al. [19] }\end{array}$ & $\begin{array}{l}20 \text { NCAA } \\
\text { division II } \\
\text { lacrosse } \\
\text { athletes }\end{array}$ & $\begin{array}{l}\text { My Fitness } \\
\text { Pal } \\
\text { Application }\end{array}$ & Accelometer & N/A & N/A & DXA & DXA & N/A & $\begin{array}{l}\text { RMR using } \\
\text { indirect } \\
\text { calorimetry. } \\
\text { Questionnaire } \\
\text { to } \\
\text { assess } \\
\text { perceived } \\
\text { rest, } \\
\text { soreness and } \\
\text { training } \\
\text { satisfaction }\end{array}$ \\
\hline 2018 & $\begin{array}{c}\text { Heikura et } \\
\text { al. [23] }\end{array}$ & $\begin{array}{l}48 \text { elite } \\
\text { distance } \\
\text { athletes } \\
21 \mathrm{M} \\
27 \mathrm{~F}\end{array}$ & $\begin{array}{c}\text { Prospective } \\
\text { dietary } \\
\text { record }\end{array}$ & Activity log & N/A & N/A & N/A & DXA & $\begin{array}{l}\text { Serum iron, } \\
\text { ferritin, } \\
\text { testosterone } \\
\text { and } \\
\text { oestradiol }\end{array}$ & $\begin{array}{c}\text { Total HB } \\
\text { mass }\end{array}$ \\
\hline 2018 & $\begin{array}{c}\text { Heikura et } \\
\text { al. [24] }\end{array}$ & $\begin{array}{l}59 \text { elite } \\
\text { distance } \\
\text { athletes } \\
24 \mathrm{M} \\
35 \mathrm{~F}\end{array}$ & $\begin{array}{l}\text { Prospective } \\
\text { dietary } \\
\text { record }\end{array}$ & Activity log & N/A & $\begin{array}{l}\begin{array}{c}\text { Metabolic } \\
\text { and }\end{array} \\
\text { reproductive } \\
\text { blood } \\
\text { hormone } \\
\text { concentrations } \\
\text { and LEAF-Q }\end{array}$ & DXA & DXA & $\begin{array}{l}\text { Oestradiol, } \\
\text { ferritin, } \\
\text { IGF-1, } \\
\text { testosterone } \\
\text { and } \\
\text { T3 }\end{array}$ & $\begin{array}{l}\text { Informal } \\
\text { questionnaire } \\
\text { of } \\
\text { injury and } \\
\text { illness } \\
\text { history }\end{array}$ \\
\hline
\end{tabular}




\begin{tabular}{|c|c|c|c|c|c|c|c|c|c|c|}
\hline Year & -Sectional and & $\begin{array}{c}\text { Participants } \\
\text { (n) }\end{array}$ & $\begin{array}{l}\text { Energy } \\
\text { Intake }\end{array}$ & $\begin{array}{c}\text { Exercise } \\
\text { Energy } \\
\text { Expenditure }\end{array}$ & $\begin{array}{l}\text { DE } \\
\text { Met }\end{array}$ & $\begin{array}{l}\text { Reproductive } \\
\text { Health } \\
\text { Is Used }\end{array}$ & BMD & $\begin{array}{c}\text { Body } \\
\text { Composition }\end{array}$ & $\begin{array}{c}\text { Biochemical } \\
\text { Parameters } \\
\text { Assessed }\end{array}$ & $\begin{array}{l}\text { Other } \\
\text { Parameters } \\
\text { Assessed }\end{array}$ \\
\hline 2018 & $\begin{array}{l}\text { Silva et al. } \\
\text { [13] }\end{array}$ & $\begin{array}{c}82 \text { children } \\
\text { and adolesc. } \\
\text { acrobatic } \\
\text { gymnasts } \\
21 \mathrm{M} \\
61 \mathrm{~F}\end{array}$ & $\begin{array}{l}\text { Prospective } \\
\text { dietary } \\
\text { record }\end{array}$ & Activity log & N/A & $\begin{array}{c}\text { Menstrual } \\
\text { history } \\
\text { questionnaire }\end{array}$ & N/A & $\begin{array}{c}\text { 3-site } \\
\text { skinfold } \\
\text { measurements }\end{array}$ & N/A & $\begin{array}{c}\text { Sleep } \\
\text { duration }\end{array}$ \\
\hline 2018 & $\begin{array}{c}\text { Zanders et } \\
\text { al. [17] }\end{array}$ & $\begin{array}{c}13 \mathrm{~F} \\
\text { collegiate } \\
\text { basketball } \\
\text { players }\end{array}$ & $\begin{array}{c}\text { Prospective } \\
\text { dietary } \\
\text { record }\end{array}$ & $\begin{array}{l}\text { HR monitor } \\
\text { and } \\
\text { accelometer }\end{array}$ & N/A & N/A & DXA & DXA & N/A & $\begin{array}{l}\text { RMR using } \\
\text { the } \\
\text { Schofield } \\
\text { equation, } \\
\text { sleep and } \\
\text { recovery } \\
\text { questionnaires }\end{array}$ \\
\hline 2017 & $\begin{array}{c}\text { Brown et al. } \\
\text { [15] }\end{array}$ & $\begin{array}{l}25 \text { F Pre- } \\
\text { professional } \\
\text { contemporary } \\
\text { dancers }\end{array}$ & $\begin{array}{l}\text { Prospective } \\
\text { weighed } \\
\text { dietary } \\
\text { record and } \\
24-\mathrm{h} \\
\text { recall }\end{array}$ & Accelometer & TFE-Q & $\begin{array}{l}\text { Menstrual } \\
\text { history } \\
\text { questionnaire }\end{array}$ & N/A & $\begin{array}{c}\text { 7-site } \\
\text { skinfold } \\
\text { measurements }\end{array}$ & N/A & $\begin{array}{l}\text { Healthier } \\
\text { dance } \\
\text { practice } \\
\text { national } \\
\text { survey }\end{array}$ \\
\hline 2017 & $\begin{array}{l}\text { Ong et al. } \\
\text { [18] }\end{array}$ & $\begin{array}{l}9 \mathrm{~F} \text { Dragon } \\
\text { boat athletes }\end{array}$ & $\begin{array}{l}\text { Prospective } \\
\text { dietary } \\
\text { record }\end{array}$ & Accelometer & N/A & N/A & N/A & $\begin{array}{c}\text { Bio- } \\
\text { impedance }\end{array}$ & N/A & N/A \\
\hline 2017 & $\begin{array}{l}\text { Silva et al. } \\
\text { [22] }\end{array}$ & $\begin{array}{c}72 \text { children } \\
\text { and adolesc. } \\
\text { M rink- } \\
\text { hockey } \\
\text { players and } \\
79 \mathrm{M} \text { ctrl }\end{array}$ & $\begin{array}{l}\text { Prospective } \\
\text { dietary } \\
\text { record }\end{array}$ & Activity log & N/A & N/A & N/A & $\begin{array}{c}\text { 2-site } \\
\text { skinfold } \\
\text { measurements }\end{array}$ & N/A & N/A \\
\hline
\end{tabular}

Abbreviations: Adolesc: adolescents; BMD: bone mineral density; Ctrl: control; DE: disordered eating; DXA: Dual-energy X-ray absorptiometry; EA: energy availability; EDE-Q: Eating Disorder Examination Questionnaire; F: female; FSH: follicle stimulating hormone; FFQ: food frequency questionnaire; HDL: high density lipoprotein; Hb; haemoglobin; HR: heart rate; IGF-1: insulin-like growth factor; LEAF-Q: Low Energy Availability in Females Questionnaire; LDL: low density lipoprotein; M: male; NCAA: national collegiate athletic association; N/A: not available; NCAA: national collegiate athletic association; RMR: resting metabolic rate; T3: tri-iodothyronine; TC: total cholesterol; TEE: total energy expenditure; TFE-Q: Three Factor Eating Questionnaire; TG: triglycerides. 
Table 3. Estimated risk of low energy availability and associated health and performance outcomes.

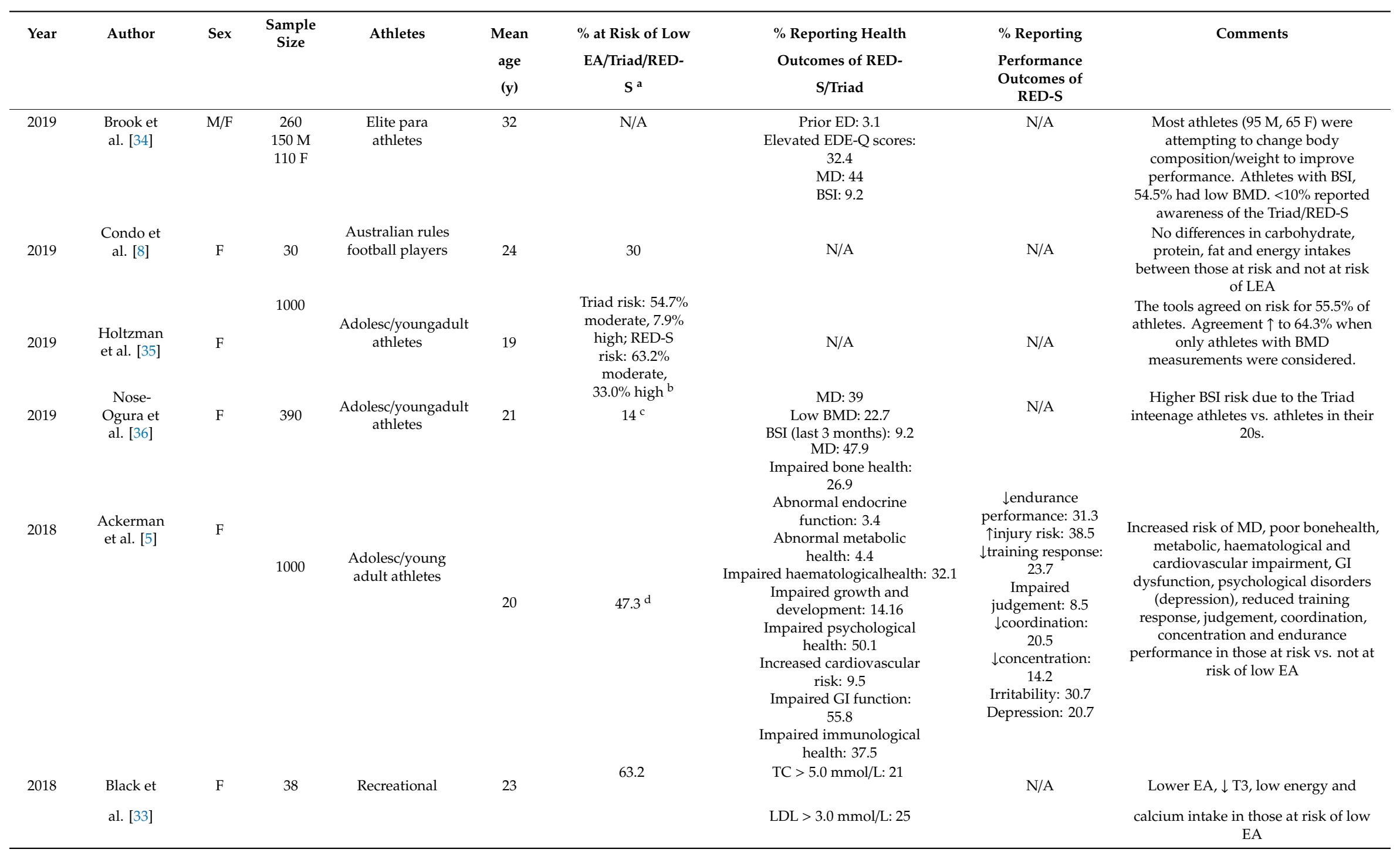




\begin{tabular}{|c|c|c|c|c|c|c|c|c|c|}
\hline Year & Author & Sex & $\begin{array}{c}\text { Sample } \\
\text { Size }\end{array}$ & Athletes & $\begin{array}{l}\text { Mean } \\
\text { age } \\
(y)\end{array}$ & $\begin{array}{c}\% \text { at Risk of Low } \\
\text { EA/Triad/RED- } \\
\mathrm{S}^{\text {a }}\end{array}$ & $\begin{array}{l}\text { \% Reporting Health } \\
\text { Outcomes of RED- } \\
\text { S/Triad }\end{array}$ & $\begin{array}{c}\% \text { Reporting } \\
\text { Performance } \\
\text { Outcomes of } \\
\quad \text { RED-S }\end{array}$ & Comments \\
\hline 2018 & $\begin{array}{l}\text { Keay et } \\
\text { al. [9] }\end{array}$ & $\mathrm{M}$ & 50 & Road cyclists & 36 & $28 \%{ }^{\mathrm{e}}$ & $\begin{array}{l}\text { Lower lumbar spine } \\
\text { BMD: } 44\end{array}$ & N/A & $\begin{array}{l}\text { Lack of load-bearing sport associated } \\
\text { with low BMD in cyclists with low } \\
\text { EA. The } 10 \text { with low EA had lower } \\
\text { testosterone levels than those } \\
\text { maintain adequate EA. Low EA } \\
\text { associated with reduced body } \\
\text { fat percentage. }\end{array}$ \\
\hline 2018 & $\begin{array}{l}\text { Logue et } \\
\text { al. [37] }\end{array}$ & $\mathrm{F}$ & 833 & $\begin{array}{l}\text { Elite, sub-elite } \\
\text { and recreational }\end{array}$ & N/A & 40 & $\begin{array}{l}\geq 22 \text { days absence from } \\
\text { training due to illness: } \\
24.2\end{array}$ & N/A & $\begin{array}{l}1.7-\text { and } 1.8 \text {-times increased risk in } \\
\text { international and provincial/inter- } \\
\text { county athletes compared to } \\
\text { recreationally active individuals }\end{array}$ \\
\hline 2018 & $\begin{array}{l}\text { Staal et } \\
\text { al. [38] }\end{array}$ & $\mathrm{M} / \mathrm{F}$ & $\begin{array}{c}40 \\
20 \mathrm{M} \\
20 \mathrm{~F}\end{array}$ & $\begin{array}{l}\text { Elite ballet } \\
\text { dancers }\end{array}$ & 25 & F: 40 & $\begin{array}{c}\text { Low C-RMR: } 100 \text { F, } 80 \\
\text { M } \\
\text { Low HB-RMR ratio: } 45 \\
\text { F,25M } \\
\text { Low DXA-RMR ratio: } 35 \\
\text { F,55M }\end{array}$ & N/A & $\begin{array}{l}\text { Large variability in suppressed RMR } \\
\text { using predictive RMR equations (M: } \\
25-80 \% \text {; F: } 35-100 \% \text { ). Cunningham } \\
\text { equation showed highest sensitivity } \\
\text { for detecting both genders at risk for } \\
\text { energy deficiency. }\end{array}$ \\
\hline 2018 & $\begin{array}{l}\text { Wilson et } \\
\text { al. [39] }\end{array}$ & M & $\begin{array}{c}21 \\
17 \mathrm{~A} \\
14 \mathrm{~S}\end{array}$ & Flat jockeys & $\begin{array}{l}\text { A: } 19 \\
\text { S: } 32\end{array}$ & N/A & N/A & N/A & $\begin{array}{l}\text { No difference in RMR or hip and } \\
\text { lumber spine BMD between groups. } \\
\text { Measured RMR did not differ from } \\
\text { predicted RMR in either group. }\end{array}$ \\
\hline 2017 & $\begin{array}{l}\text { Drew et } \\
\text { al. [40] }\end{array}$ & $\mathrm{M} / \mathrm{F}$ & $\begin{array}{l}132 \\
47 \mathrm{M} \\
85 \mathrm{~F}\end{array}$ & $\begin{array}{l}\text { Elite Olympic } \\
\text { athletes }\end{array}$ & $\begin{array}{l}\text { M: } 26 \\
\text { F: } 24\end{array}$ & 40 & N/A & N/A & $\begin{array}{l}\text { Higher odds of reporting URTI } \\
(\mathrm{OR}=3.8) \text {, bodily aches }(\mathrm{OR}=5.8), \mathrm{GI} \\
\text { disturbances }(\mathrm{OR}=3.8) \text { and head } \\
\text { symptoms (OR }=4.4) \text { in those at risk } \\
\text { of low EA. }\end{array}$ \\
\hline 2017 & $\begin{array}{l}\text { Sygo et } \\
\text { al. [41] }\end{array}$ & F & 13 & Elite sprinters & 21 & $\begin{array}{l}\text { Pre-training } \\
\text { season: } 23 \text { Post- } \\
\text { training season: } \\
39\end{array}$ & $\begin{array}{c}\text { Pre-training season: } \\
\text { BMD: } 8 \text { RMR: } 15 \text { FSH: } \\
15 \\
\text { Post-training season: } \\
\text { BMD: } 15 \text { RMR: } 8 \\
\text { oestradiol: } 31 \text { LH: } 23 \\
\text { FSH: } 15\end{array}$ & N/A & $\begin{array}{c}\text { Primary low EA indicators: a LEAF- } \\
\text { Q score }>8 \text {; RMR }<29 \mathrm{kcal} / \mathrm{kg} \text { FFM, } \\
\text { low oestradiol, FSH or LH or a BMD } \\
\text { of }<1.09 \mathrm{~g} / \mathrm{cm}^{2}\end{array}$ \\
\hline
\end{tabular}

Abbreviations: A: apprentice flat jockey; Adolesc: adolescents; BEDA-Q: brief eating disorder in athletes questionnaire; BMD: bone mineral density; BP: blood pressure; BSI: bone stress injury; C-RMR ratio: the ratio between measured and predicted RMR using Cunningham equation; DE: disordered eating; DXA-RMR ratio: the ratio between measured and predicted RMR using different tissue compartments from DXA; EA: energy availability; ED: eating disorder; EDE-Q: eating disorder examination questionnaire; EDI-3: eating disorder inventory; ESP: eating disorder screen for primary care; F: female; FFM: fat free mass; FSH: follicle stimulating hormone; GI: gastrointestinal; HB-RMR ratio: the ratio between measured and predicted RMR using the Harris-Benedict equation; LEAF-Q: low energy availability in females questionnaire; LH: luteinising hormone; M: male; MD: menstrual dysfunction; OR: odds ratio; \%: percentage; RED-S: relative energy deficiency in sport; RED-S CAT: Relative Energy Deficiency in Sports Clinical Assessment Tool; REDS-outcomes; assessed conditions related to RED-S included blood pressure, eating disorder inventory scores and bone mineral density: RMR: resting metabolic rate; S: senior flat jockey: Triad CRA: Female Athlete Triad Cumulative Risk A Assessment $\downarrow$ dect self-reported current or history of ED or DE e Risk of RED-S assessed using a sport-specific questionnaire and clinical interview (SEAQ-I). 
Furthermore, a low ratio between measured and predicted resting metabolic rate (RMR) is an acknowledged marker of low EA (RMR ratio < 0.90) [27]. Associations between suppressed RMR and elevated low EA risk scores in female ballet dancers and with higher training volume in male ballet dancers have recently been observed [38]. These results indicate low RMR ratio as a potential surrogate marker for low EA. However, the prevalence of suppressed RMR ratio using dual-energy X-ray (DXA) and different RMR predictive equations such as the Harris-Benedict and Cunningham equations showed large variability in both male and female professional ballet dancers, ranging from $25 \%$ to $80 \%$ and $35 \%$ to $100 \%$ in males and females, respectively [38]. Thus, the identification of athletes at risk of low EA varies greatly depending on the predictive equation used. A recent review of the validity of RMR predictive equations to assess low EA in athletes emphasized that predictive equations that do not have FFM incorporated within the algorithm are unsuitable for use in athletes and that accurate RMR laboratory protocols are essential when monitoring EA [42]. Thus, the development of a reliable RMR laboratory protocol in athletes is needed before measured RMR or the RMR ratio can reliably be used to diagnose low EA $[27,38,43]$.

\subsection{Low Energy Availability Risk}

The risk of low EA has been investigated using surrogate markers or self-reported symptoms of low EA in various athlete populations including elite para-athletes [34], adolescent/young adult [35,36] and Olympic athletes [40], female sprinters [41], male jockeys [39] and recreationally active individuals [33,37], and ranges from $14 \%$ to $63 \%$ (Table 3). The largest cross-sectional study investigating self-reported health and performance outcomes linked to low EA and RED-S [5] placed emphasis on including body systems beyond reproductive function and bone health such as metabolic, haematological, psychological and cardiovascular health and gastrointestinal function. The authors acknowledged that associations identified in their study (Table 3) were based on self-reported data and highlight the need to investigate the health and performance components of RED-S in a controlled setting, whereby low EA is measured under strict conditions in a laboratory setting, to understand causative pathways [5]. Another cross-sectional study supports associations between low EA risk and self-reported medical illness [40]. Moreover, associations have been described between low EA risk and stress fractures, absence from training for $>22$ days due to illness and reported adherence to a gluten-free diet [37]. These findings suggest that those at risk of low EA can present with symptoms other than those traditionally expected e.g., menstrual irregularities, and highlight the complexity of identifying individuals at risk [40]. The LEAF-Q enables early recognition of active females at risk of low EA by evaluating the presence of symptoms associated with low EA, such as menstrual and gastrointestinal dysfunction, injury history, as well as oral contraceptive use [30]. The LEAF-Q is an easily administered questionnaire that has been validated for use in athletic endurance-trained females [30]. As such, it offers a validated method to investigate risk of low EA in large, exercising cohorts and alleviates some of the challenges associated with directly measuring EA [44]. In the measurement of the components of the EA equation, significant errors of reliability (e.g., variation in the time and techniques used to estimate EA) and validity (e.g., under/over estimation of EI and/or EEE) can occur [45]. Although low EA risk has been investigated in elite male cyclists [9] using non-validated tools such as a sport-specific questionnaire and clinical interview (SEAQ-I) [9], a screening questionnaire for male athletes similar to the LEAF-Q is required and is currently being developed (the Low Energy Availability in Male's Questionnaire (LEAM-Q) [2]. Following its development and validation, it is anticipated that potential risk of low EA will be identified in a range of athletic male populations.

\subsubsection{Eating Disorders and Exercise Addiction}

Disordered eating behaviours and eating disorders are known to occur frequently in elite female athletes, particularly among those competing in weight class or leanness-demanding sports [46]. These conditions are associated with perfectionism as well as compulsive exercise behaviour, coupled with the inability to reduce training load [47] and can lead to unfavourable outcomes such as injury and 
emotional distress [47]. Recent research describes how compulsive exercise behaviour is associated with symptoms of disordered eating behaviour, perfectionism and obsessive-compulsive characteristics in long-distance runners of both sexes [48]. We encourage further exploration of the relationship between compulsive exercise behaviour and RED-S, since individuals demonstrating compulsive exercise behaviour may be at greater risk of the negative health outcomes associated with low EA [48].

Although most research into RED-S has been conducted in females, a recent study investigated associations between compulsive exercise behaviour, disordered eating symptoms and biomarkers of RED-S in trained male cyclists, triathletes and long-distance runners [49]. Using the Eating Disorder Examination Questionnaire (EDE-Q), higher total exercise dependence scores were associated with disordered eating symptoms. Participants with higher exercise dependence scores trained more frequently ( $\sim 11 \mathrm{~h} /$ week) compared to those with lower scores ( $\sim 8 \mathrm{~h} /$ week). Furthermore, participants with higher scores did not adjust their energy intake to meet increased energy needs, resulting in more pronounced low EA. This was associated with higher cortisol levels compared to those with lower exercise dependence scores [49]. Higher subscale exercise dependence scores were also associated with lower blood glucose, lower testosterone: cortisol ratio and higher cortisol: insulin ratio [49]. This suggests an association between biomarkers of RED-S and compulsive exercise behaviour. Although these findings currently relate only to endurance athletes, determining whether these associations exist in highly-trained athletes competing, in particular, in weight sensitive and team sports, represents an interesting area for future investigation.

Psychological factors such as stress, anxiety and depression can result in or contribute to disordered eating behaviour and low EA in athletes [1]. Moreover, it has been suggested that compulsive exercise behaviour could increase vulnerability to negative health and performance outcomes [48]. Despite the lack of conclusive evidence, mainly due to the absence of studies investigating associations between compulsive exercise, disordered eating behaviour symptoms and biomarkers of RED-S, there appears to be an opportunity to improve athlete health and performance. This may be possible through screening for potential underlying causes of low EA such as compulsive exercise, in conjunction with validated disordered eating behaviour and low EA risk questionnaires. Screening for compulsive exercise should increase awareness of the psychological factors underpinning and contributing to low EA and its associated consequences such as poor mental health. More precisely, identifying the relationship between compulsive exercise, disordered eating behaviour and RED-S in various sports and groups of athletes (e.g., gender, performance level and age) is recommended. Nevertheless, self-reported data need to be interpreted with caution as they may be heavily influenced by athletes' perceptions, experiences and recollections, thus, further emphasising the need to use well-validated questionnaires to investigate outcomes associated with LEA in the athletic population.

\subsubsection{Exercise Hypogonadal Male Condition}

The Triad continuum, whereby a female can advance from optimal health status [optimal EA, normal menstrual function and good bone health] to a diseased state [low EA (with and without an eating disorder), menstrual dysfunction and low bone mineral density] at any point throughout her life course, has been extensively investigated over the last 30 years [50-52]. Rigorously executed studies have identified low EA, not the stress of exercise, as the underlying mechanism causing disruption to the female hypothalamic-pituitary-gonadal axis [51,53]. Further evidence suggests that endurance exercising males can develop a similar suppression of the reproductive function known as exercise hypogonadal male condition (EHMC) [54] (Figure 1). In EHMC, disruption to the male hypothalamic-pituitary-gonadal axis has been hypothesised, given that luteinising hormone and testosterone levels are suppressed (Figure 2 Left), just as in females with functional hypothalamic oligomenorrhea or amenorrhea (Figure 2 Right). In male athletes, associations between higher levels of intensive endurance training and reductions in testosterone and libido [6,54] have been observed without assessment of EA, and the underlying mechanisms remain unclear. Further research in a controlled setting (i.e., low EA is measured under strict conditions in a laboratory setting) is warranted 
to understand the causative pathways behind disruption to the male hypothalamic-pituitary-gonadal axis. Furthermore, accurately determining the reliability of testosterone levels as a potential indicator of low EA or RED-S and the impact of low testosterone levels on other testosterone-dependent physiological processes warrants thorough investigation.

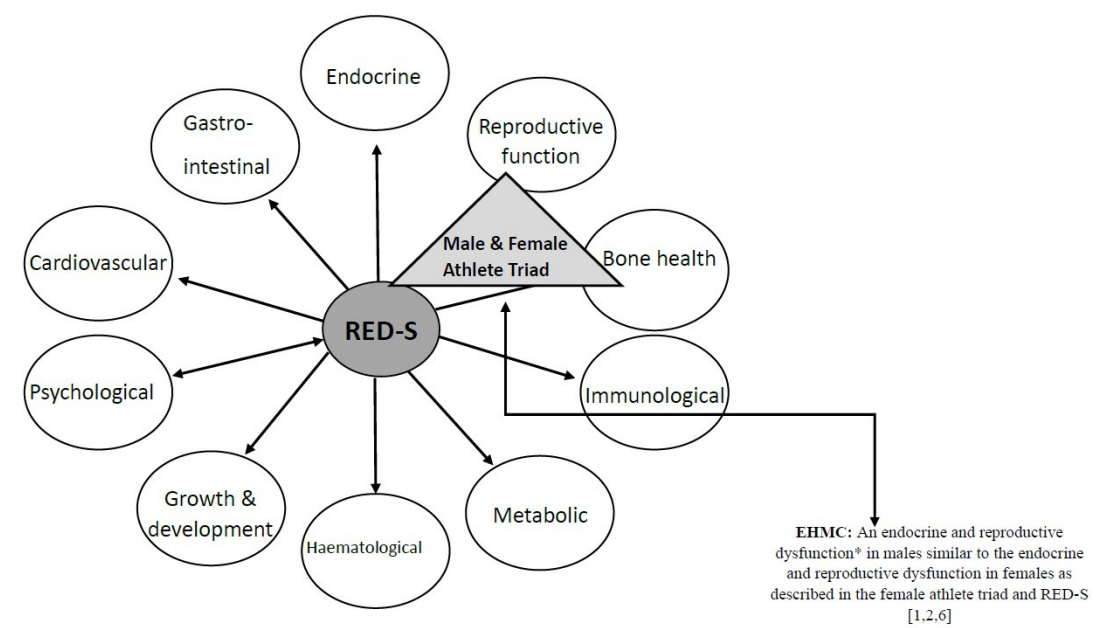

Figure 1. Adapted from the relative energy deficiency in sport health model [1,2] with the inclusion of the male and female athlete triad and the exercise-hypogonadal male condition [6]. Abbreviations: EHMC: exercise-hypogonadal male condition; RED-S: relative energy deficiency in sport *the exact physiological mechanism inducing the reduction of testosterone in men is currently unclear; it is postulated to be a dysfunction within the hypothalamic-pituitary-testicular regulatory axis.

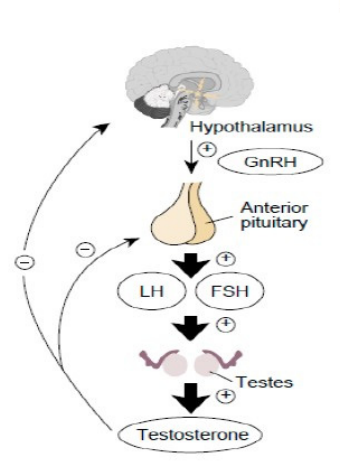

Reproductive components of the neurpendocrine system*

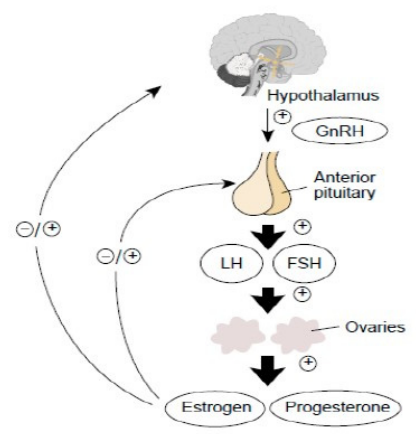

Figure 2. Male and female hypothalamic-pituitary-gonadal axes. Reprinted with permission: Artoria2e5 [CC BY 4.0 (https://creativecommons.org/licenses/by/4.0/) *the reproductive components of the neuroendocrine system in the body are extremely sensitive to Low Energy Availability (LEA) in females [1,2] and the stress of exercise in males [6].

\section{Within-Day Energy Deficiency in Athletes}

Recent research into within-day energy balance and within-day energy deficiency, whereby energy intake and exercise energy expenditure are assessed in 1-h intervals, may provide greater insight into real-time changes that are indicative of the endocrine responses associated with variations in EA and may help to identify markers of energy deficiency $[55,56]$. To date, two studies have examined within-day energy deficiency, one in male [56] and the other in female [55] endurance athletes. In the male study, the relationship between within-day energy deficiency and RMR was explored [56]. Despite similar EA, athletes identified with suppressed RMR spent more time (21 vs. $11 \mathrm{~h}$ ) with an energy deficit exceeding $400 \mathrm{kcal}$ across a 24-h period. Within-day energy deficiency was associated with higher blood cortisol level and lower testosterone: cortisol ratio [56]. In the female study, within-day energy deficiency was also associated with higher cortisol levels as well as with menstrual dysfunction, 
lower oestradiol and RMR ratio [55]. Furthermore, despite similar EA, female endurance athletes with menstrual dysfunction spent more time ( $22 \mathrm{vs} .18 \mathrm{~h}$ ) in an energy deficient state exceeding $300 \mathrm{kcal}$ compared to eumenorrheic athletes [55]. A positive association between within-day energy deficiency and more frequent meals was also observed. These findings contradict the commonly held belief that higher meal frequency may be necessary for some athletes to attain energy balance and prevent within-day energy deficiency [57]. Consumption of high fibre and low energy-dense foods were previously reported in endurance females with menstrual dysfunction [58]; thus, the energy density of food consumed at mealtimes needs careful consideration to improve within-day energy balance in females with menstrual dysfunction. Other dietary factors such as diet quality and differing attitudes towards food groups need to be investigated when assessing for risk of low EA.

In summary, the viability of measuring within-day energy deficiency and its ability to detect athletes at risk of negative health outcomes associated with low EA is unclear. Questions around appropriate assessment of within-day energy deficiency and the time periods over which it should be assessed impede the quality of research in this area. Further research, especially in female athletes, represents an interesting area for future investigation.

\section{Low Energy Availability and Sports Performance}

While low EA influences many body systems, for example, reproductive system suppression and menstrual cycle disruption as a mechanism to conserve energy, it is important to note other hormonal pathways are altered, resulting in numerous interrelated endocrine-derived physiological consequences [50,59]. These include increased cortisol levels and reduced triiodothyronine (T3), luteinizing hormone ( $\mathrm{LH})$ pulsatility and hypoestrogenism [50,59]. Low EA-related menstrual dysfunction is associated with increased bone stress injury risk which can impair training and competition availability [60]. Thus, low EA may be a contributor to poor sports performance due to associated detrimental endocrine effects [61]. A decrease in neuromuscular performance, assessed using isokinetic dynamometry, was observed in elite endurance athletes with menstrual dysfunction in contrast to eumenorrheic endurance athletes [62]. Furthermore, the decreased neuromuscular performance was associated with lower FFM in the leg, glucose, oestrogen, T3, and elevated cortisol [62]. While these findings are unable to provide sufficient evidence of a causal link between these biomarkers and performance, the interrelationship is biologically possible. The study authors hypothesized that a consistently low blood glucose may lead to increased cortisol and reduced T3, in addition to lower muscle mass in the long term, all of which have been associated with reduced neuromuscular performance [62]. Furthermore, these results support previous literature that indicates that physiological manifestations of low EA, such as menstrual dysfunction in female athletes, negatively impact on sporting performance $[63,64]$.

One study, which did not assess EA but instead investigated associations between aspects of endurance exercise training and sexual libido in endurance training males, concluded that libido scores are associated with the duration and intensity of training [6]. These findings indicate that physiological adaptations may occur within males but reliance on self-reported measures limits the interpretation and generalisability of study results [6]. Nonetheless, these preliminary findings indicate that the amount and intensity of endurance training as potential contributing factors to low libido in males needs consideration. Further research is necessary to better understand the impact of low libido on sports performance.

\section{Knowledge of Low Energy Availability and Relative Energy Deficiency in Sport}

Screening and interventions for low EA risk are needed to protect athlete health and performance. Coaches can play a pivotal role in the early identification of athletes at risk of low EA. However, there is little research exploring the knowledge coaches have on this topic. A recent cross-sectional survey that investigated knowledge of the Triad and RED-S among head athletic trainers at National Collegiate Athletic Association (NCAA) member institutions reported that almost all principal athletic trainers 
(98.6\%) were aware of the Triad [10]. Only one-third (33\%) were aware of RED-S [10]. Furthermore, $60 \%$ and $71 \%$ of principal athletic trainers reported that athletes were screened for eating disorders and menstrual irregularities respectively. Those athletes in need of multidisciplinary team support (e.g., athletes identified with menstrual dysfunction or bone stress injury) occurred at division I institutions compared to those in division II or III. A major limitation of this study is that only one third of the principal athletic trainers at NCAA member institutions responded to the survey. It may be that interest and knowledge of the Triad and RED-S was greater in study participants than in those who failed to respond.

Another study investigating risk of low EA and sports nutrition knowledge among female Australian Rules football players reported that $30 \%$ of participants were at risk of low EA [8]. Nutrition knowledge of participants was measured using the Sports Nutrition Knowledge Questionnaire [65]. Players answered $\sim 55 \%$ of the questions correctly, with the lowest scores observed for responses in the supplement section [8]. Furthermore, an intervention study investigating the effect of a 6-month nutrition education program for male athletes who were at risk of RED-S, reported positive results on bone health and race performance [9]. These findings suggest that nutritional education programs, including specific information on low EA, its associated short and long-term health implications and dietary supplement use, may improve athlete health and performance outcomes. Education on RED-S for athletes and coaches is necessary to encourage screening for and early identification of athletes at risk of low EA. Multidisciplinary healthcare professional input to ensure appropriate interpretation of the results of screening questionnaires and implementation of interventions is required.

A recent intervention investigated changes in Triad knowledge among female high school athletes $(n=89)$ after participation in a number of brief $(10-\mathrm{min})$ Triad educational videos [11]. The educational videos featured (1) a registered dietitian who defined the aetiology and progression of the Triad, and who provided nutritional strategies that may decrease its risk, (2) a former collegiate athlete who shared personal experiences of overcoming Triad-associated problems and, (3) a collegiate coach who shared insights into negative body image and pressure to achieve a specific bodyweight or appearance [11]. Knowledge about consequences of the Triad (for example, menstrual irregularity increases stress fracture risk; low energy intake is negatively associated with menstrual function and bone health) was identified in most participating athletes $(\geq 89 \%)$ [11]. These findings suggest that educational videos may be an effective method of improving athlete knowledge of the Triad. Whether improved knowledge is associated with behavioural change in athletes with low EA (for example, athletes increasing their energy intake) represents an important area for investigation.

\section{Conclusions}

This article provides a synthesis of the research conducted on RED-S since our previously published review [7]. This is important given the number of studies conducted over the period. Detection of low EA by identifying physiological symptoms and measuring biomarkers associated with the condition has replaced the use of weighed dietary and exercise logs and heart rate monitors or accelerometers. This may facilitate better exploration of the health and performance outcomes associated with low EA. As the majority of studies are cross-sectional in design and cannot show causality, future longitudinal studies with sufficient numbers of those at risk are required to properly understand the consequences of low EA. The poor understanding by athletes and coaches of low EA and its potential health and performance consequences emphasizes the need for further research in this area. Education to increase awareness and to implement dietary and training interventions, in particular, for athletes engaged in intensive training is also vital.

Key points: Until 2015, low energy availability (EA) was predominantly identified using weighed dietary and exercise logs in combination with heart rate monitors or accelerometers [7]. Research published in the past four years has established that low EA is more easily and accurately identified by the use of surrogate markers including suppressed resting RMR [27,38,43] and validated questionnaires that screen for a drive for thinness and physiological symptoms associated with low 
EA [30]. Understanding athletes' and coaches' knowledge and perspectives on low EA and its health and performance consequences is required [10]. Few education initiatives exist to improve athlete understanding of low EA and its associated health and performance consequences [11]. The development and implementation of sports nutrition programmes to increase awareness and improve knowledge of EA and within-day energy balance, as well as sports nutrition treatment strategies for athletes at risk of low EA, are warranted.

Author Contributions: D.M.L., C.A.C., A.M., S.M.M., E.D., S.-J.M.D., and M.H. were involved in the review planning. D.L. was responsible for drafting the review paper. D.L. drafted and designed the tables/figures. C.A.C., A.M., S.M.M., E.D., S.M.M., and M.H. contributed to writing the review paper. All authors discussed the review findings, provided feedback and approved the manuscript prior to submission. All authors have read and agreed to the published version of the manuscript.

Funding: This research is funded by the Irish Research Council (IRC) and Sport Ireland (grant number: EPS-PG-2015-99).

Acknowledgments: The authors would like to acknowledge the full support and collaboration of the Irish Research Council and Sport Ireland Institute.

Conflicts of Interest: Danielle Logue, Sharon Madigan, Anna Melin, Eamonn Delahunt, Mirjam Heinen, Sarah-Jane McDonnell and Clare Corish declare that they have no conflicts of interest relevant to the content of this review.

\section{References}

1. Mountjoy, M.; Sundgot-Borgen, J.; Burke, L.; Carter, S.; Constantini, N.; Lebrun, C.; Meyer, N.; Sherman, R.; Steffen, K.; Budgett, R.; et al. The IOC consensus statement: Beyond the female athlete triad-relative energy deficiency in sport (RED-S). Br. J. Sports Med. 2014, 48, 491-497. [CrossRef] [PubMed]

2. Mountjoy, M.; Sundgot-Borgen, J.K.; Burke, L.M.; Ackerman, K.E.; Blauwet, C.; Constantini, N.; Lebrun, C.; Lundy, B.; Melin, A.K.; Meyer, N.L.; et al. IOC consensus statement on relative energy deficiency in sport (RED-S): 2018 update. Br. J. Sports Med. 2018, 52, 687-697. [CrossRef] [PubMed]

3. Loucks, A.B.; Kiens, B.; Wright, H.H. Energy availability in athletes. J. Sports Sci. 2011, 29 (Suppl. 1), S7-S15. [CrossRef] [PubMed]

4. Raysmith, B.P.; Drew, M.K. Performance success or failure is influenced by weeks lost to injury and illness in elite Australian track and field athletes: A 5-year prospective study. J. Sci. Med. Sport 2016, 19, 778-783. [CrossRef] [PubMed]

5. Ackerman, K.E.; Holtzman, B.; Cooper, K.M.; Flynn, E.F.; Bruinvels, G.; Tenforde, A.S.; Popp, K.L.; Popp, K.L.; Simpkin, A.J.; Parziale, A.L. Low energy availability surrogates correlate with health and performance consequences of Relative Energy Deficiency in Sport. Br. J. Sports Med. 2019, 53, 628-633. [CrossRef] [PubMed]

6. Hackney, A.C.; Lane, A.R.; Register-Mihalik, J.; O'Leary, B.C. Endurance exercise training and male sexual libido. Med. Sci. Sports Exerc. 2017, 49, 1383-1388. [CrossRef] [PubMed]

7. Logue, D.; Madigan, S.M.; Delahunt, E.; Heinen, M.; McDonnell, S.J.; Corish, C.A. Low energy availability in athletes: A review of prevalence, dietary patterns, physiological health, and sports performance. Sports Med. 2018, 48, 73-96. [CrossRef]

8. Condo, D.; Lohman, R.; Kelly, M.; Carr, A. Nutritional intake, sports nutrition knowledge and energy availability in female australian rules football players. Nutrients 2019, 11, 971. [CrossRef]

9. Keay, N.; Francis, G.; Entwistle, I.; Hind, K. Clinical evaluation of education relating to nutrition and skeletal loading in competitive male road cyclists at risk of relative energy deficiency in sports (RED-S): 6-month randomised controlled trial. BMJ Open Sport Exerc. Med. 2019, 5, e000523. [CrossRef]

10. Kroshus, E.; DeFreese, J.D.; Kerr, Z.Y. Collegiate athletic trainers' knowledge of the female athlete triad and relative energy deficiency in sport. J. Athl Train. 2018, 53, 51-59. [CrossRef]

11. Krick, R.L.; Brown, A.F.; Brown, K.N. Increased female athlete triad knowledge following a brief video educational intervention. J. Nutr. Educ. Behav. 2019, 51, 1126-1129. [CrossRef] [PubMed]

12. Harbour, R.; Miller, J. A new system for grading recommendations in evidence based guidelines. BMJ 2001, 323, 334-336. [CrossRef] [PubMed] 
13. Silva, M.G.; Silva, H.H.; Paiva, T. Sleep duration, body composition, dietary profile and eating behaviours among children and adolescents: A comparison between Portuguese acrobatic gymnasts. Eur. J. Pediatr. 2018, 177, 815-825. [CrossRef] [PubMed]

14. Civil, R.; Lamb, A.; Loosmore, D.; Ross, L.; Livingstone, K.; Strachan, F.; Dick, J.R.; Stevenson, E.J.; Brown, M.A.; Witard, O.C. Assessment of dietary intake, energy status, and factors associated with RED-S in vocational female ballet students. Front Nutr. 2018, 5, 136. [CrossRef] [PubMed]

15. Brown, M.A.; Howatson, G.; Quin, E.; Redding, E.; Stevenson, E.J. Energy intake and energy expenditure of pre-professional female contemporary dancers. PLoS ONE 2017, 12, e0171998. [CrossRef] [PubMed]

16. Costa, P.B.; Richmond, S.R.; Smith, C.R.; Currier, B.; Stecker, R.A.; Gieske, B.T.; Kemp, K.; Witherbee, K.E.; Kerksick, C.M. Physiologic, metabolic, and nutritional attributes of collegiate synchronized swimmers. Int. J. Sports Physiol. Perform. 2019, 14, 658-664. [CrossRef]

17. Zanders, B.R.; Currier, B.S.; Harty, P.S.; Zabriskie, H.A.; Smith, C.R.; Stecker, R.A.; Richmond, S.R.; Jagim, A.R.; Kerksick, C.M. Changes in energy expenditure, dietary intake, and energy availability across an entire collegiate women's basketball season. J. Strength Cond. Res. 2018, 17. [CrossRef]

18. Ong, J.L.; Brownlee, I.A. Energy expenditure, availability, and dietary intake assessment in competitive female dragon boat athletes. Sports 2017, 5, 45. [CrossRef]

19. Zabriskie, H.A.; Currier, B.S.; Harty, P.S.; Stecker, R.A.; Jagim, A.R.; Kerksick, C.M. Energy status and body composition across a collegiate women's lacrosse season. Nutrients 2019, 11, 470. [CrossRef]

20. Cherian, K.S.; Sainoji, A.; Nagalla, B.; Yagnambhatt, V.R. Energy balance coexists With disproportionate macronutrient consumption across pretraining, during training, and posttraining among indian junior soccer players. Pediatr. Exerc. Sci. 2018, 30, 506-515. [CrossRef]

21. Braun, H.; von Andrian-Werburg, J.; Schänzer, W.; Thevis, M. Nutrition status of young elite female german football players. Pediatr. Exerc. Sci. 2017, 30, 157-167. [CrossRef] [PubMed]

22. Silva, M.G.; Silva, H.H. Comparison of body composition and nutrients' deficiencies between Portuguese rink-hockey players. Eur. J. Pediatr. 2017, 176, 41-50. [CrossRef] [PubMed]

23. Heikura, I.A.; Burke, L.M.; Bergland, D.; Uusitalo, A.L.T.; Mero, A.A.; Stellingwerff, T. Impact of energy availability, health, and sex on hemoglobin-mass responses following live-high-train-high altitude training in elite female and male distance athletes. Int. J. Sports Physiol. Perform. 2018, 13, 1090-1096. [CrossRef] [PubMed]

24. Heikura, I.A.; Uusitalo, A.L.T.; Stellingwerff, T.; Bergland, D.; Mero, A.A.; Burke, L.M. Low energy availability is difficult to assess but outcomes have large impact on bone injury rates in elite distance athletes. Int. J. Sport Nutr. Exerc. Metab. 2018, 28, 403-411. [CrossRef] [PubMed]

25. McCormack, W.P.; Shoepe, T.C.; LaBrie, J.; Almstedt, H.C. Bone mineral density, energy availability, and dietary restraint in collegiate cross-country runners and non-running controls. Eur. J. Appl. Physiol. 2019, 119, 1747-1756. [CrossRef] [PubMed]

26. Koehler, K.; Achtzehn, S.; Braun, H.; Mester, J.; Schaenzer, W. Comparison of self-reported energy availability and metabolic hormones to assess adequacy of dietary energy intake in young elite athletes. Appl. Physiol. Nutr. Metab. 2013, 38, 725-733. [CrossRef]

27. Melin, A.; Tornberg, Å.B.; Skouby, S.; Møller, S.S.; Sundgot-Borgen, J.; Faber, J.; Sidelmann, J.J.; Aziz, M.; Sjödin, A. Energy availability and the female athlete triad in elite endurance athletes. Scand. J. Med. Sci. Sports 2015, 25, 610-622. [CrossRef]

28. Williams, N.I.; Leidy, H.J.; Hill, B.R.; Lieberman, J.L.; Legro, R.S.; De Souza, M.J. Magnitude of daily energy deficit predicts frequency but not severity of menstrual disturbances associated with exercise and caloric restriction. Am. J. Physiol. Endocrinol. Metab. 2015, 308, E29-E39. [CrossRef]

29. Reed, J.L.; De Souza, M.J.; Mallinson, R.J.; Scheid, J.L.; Williams, N.I. Energy availability discriminates clinical menstrual status in exercising women. J. Int. Soc. Sports Nutr. 2015, 12, 11. [CrossRef]

30. Melin, A.; Tornberg, A.B.; Skouby, S.; Faber, J.; Ritz, C.; Sjödin, A.; Sundgot-Borgen, J. The LEAF questionnaire: A screening tool for the identification of female athletes at risk for the female athlete triad. Br. J. Sports Med. 2014, 48, 540-545. [CrossRef]

31. Joy, E.; De Souza, M.J.; Nattiv, A.; Misra, M.; Williams, N.I.; Mallinson, R.J.; Gibbs, J.C.; Olmsted, M.; Goolsby, M.; Matheson, G. 2014 female athlete triad coalition consensus statement on treatment and return to play of the female athlete triad. Curr. Sports Med. Rep. 2014, 13, 219-232. [CrossRef] [PubMed] 
32. Mountjoy, M.; Sundgot-Borgen, J.; Burke, L.; Carter, S.; Constantini, N.; Lebrun, C.; Meyer, N.; Sherman, R.; Steffen, K.; Budgett, R.; et al. RED-S CAT. Relative Energy Deficiency in Sport (RED-S) Clinical Assessment Tool (CAT). Br. J. Sports Med. 2015. [CrossRef] [PubMed]

33. Black, K.; Slater, J.; Brown, R.C.; Cooke, R. Low energy availability, plasma lipids, and hormonal profiles of recreational athletes. J. Strength Cond. Res. 2018, 32, 2816-2824. [CrossRef] [PubMed]

34. Brook, E.M.; Tenforde, A.S.; Broad, E.M.; Matzkin, E.G.; Yang, H.Y.; Collins, J.E.; Blauwet, C.A. Low energy availability, menstrual dysfunction, and impaired bone health: A survey of elite para athletes. Scand. J. Med. Sci. Sports 2019, 29, 678-685. [CrossRef]

35. Holtzman, B.; Tenforde, A.S.; Parziale, A.L.; Ackerman, K.E. Characterization of risk quantification differences using female athlete triad cumulative risk assessment and relative energy deficiency in sport clinical assessment tool. Int. J. Sport Nutr. Exerc. Metab. 2019, 29, 569-575. [CrossRef]

36. Nose-Ogura, S.; Yoshino, O.; Dohi, M.; Kigawa, M.; Harada, M.; Hiraike, O.; Onda, T.; Osuga, Y.; Fujii, T.; Saito, S. Risk factors of stress fractures due to the female athlete triad: Differences in teens and twenties. Scand. J. Med. Sci. Sports 2019, 29, 1501-1510. [CrossRef]

37. Logue, D.M.; Madigan, S.M.; Heinen, M.; McDonnell, S.J.; Delahunt, E.; Corish, C.A. Screening for risk of low energy availability in athletic and recreationally active females in Ireland. Eur. J. Sport Sci. 2018, 19, 112-122. [CrossRef]

38. Staal, S.; Sjödin, A.; Fahrenholtz, I.; Bonnesen, K.; Melin, A.K. Low RMRratio as a surrogate marker for energy deficiency, the choice of predictive equation vital for correctly identifying male and female ballet dancers at risk. Int. J. Sport Nutr. Exerc. Metab. 2018, 28, 412-418. [CrossRef]

39. Wilson, G.; Martin, D.; Morton, J.P.; Close, G.L. Male flat jockeys do not display deteriorations in bone density or resting metabolic rate in accordance with race riding experience: Implications for RED-S. Int. J. Sport Nutr. Exerc. Metab. 2018, 28, 434-439. [CrossRef]

40. Drew, M.K.; Vlahovich, N.; Hughes, D.; Appaneal, R.; Peterson, K.; Burke, L.; Lundy, B.; Toomey, M.; Watts, D.; Lovell, G.; et al. A multifactorial evaluation of illness risk factors in athletes preparing for the Summer Olympic Games. J. Sci. Med. Sport 2017, 20, 745-750. [CrossRef]

41. Sygo, J.; Coates, A.M.; Sesbreno, E.; Mountjoy, M.L.; Burr, J.F. Prevalence of indicators of low energy availability in elite female sprinters. Int. J. Sport Nutr. Exerc. Metab. 2018, 28, 490-496. [CrossRef] [PubMed]

42. Schofield, K.L.; Thorpe, H.; Sims, S.T. Resting metabolic rate prediction equations and the validity to assess energy deficiency in the athlete population. Exp. Physiol. 2019, 104, 469-475. [CrossRef] [PubMed]

43. Bone, J.L.; Burke, L.M. No difference in young adult athletes' resting energy expenditure when measured under inpatient or outpatient conditions. Int. J. Sport Nutr. Exerc. Metab. 2018, 28, 464-467. [CrossRef] [PubMed]

44. Mountjoy, M.L.; Burke, L.M.; Stellingwerff, T.; Sundgot-Borgen, J. Relative energy deficiency in sport: The tip of an iceberg. Int. J. Sport Nutr. Exerc. Metab. 2018, 28, 313-315. [CrossRef] [PubMed]

45. Burke, L.M.; Lundy, B.; Fahrenholtz, I.L.; Melin, A.K. Pitfalls of conducting and interpreting estimates of energy availability in free-living athletes. Int. J. Sport Nutr. Exerc. Metab. 2018, 28, 350-363. [CrossRef] [PubMed]

46. Sundgot-Borgen, J.; Torstveit, M.K. Prevalence of eating disorders in elite athletes is higher than in the general population. Clin. J. Sport Med. 2004, 14, 25-32. [CrossRef]

47. Lichtenstein, M.B.; Hinze, C.J.; Emborg, B.; Thomsen, F.; Hemmingsen, S.D. Compulsive exercise: Links, risks and challenges faced. Psychol. Res. Behav. Manag. 2017, 10, 85-95. [CrossRef]

48. Turton, R.; Goodwin, H.; Meyer, C. Athletic identity, compulsive exercise and eating psychopathology in long-distance runners. Eat Behav. 2017, 26, 129-132. [CrossRef]

49. Torstveit, M.K.; Fahrenholtz, I.L.; Lichtenstein, M.B.; Stenqvist, T.B.; Melin, A.K. Exercise dependence, eating disorder symptoms and biomarkers of Relative Energy Deficiency in Sports (RED-S) among male endurance athletes. BMJ Open Sport Exerc. Med. 2019, 5, e000439. [CrossRef]

50. Nattiv, A.; Loucks, A.B.; Manore, M.M.; Sanborn, C.F.; Sundgot-Borgen, J.; Warren, M.P. American college of sports medicine position stand. The female athlete triad. Med. Sci. Sports Exerc. 2007, 39, 1867-1882.

51. Loucks, A.B.; Verdun, M.; Heath, E.M. Low energy availability, not stress of exercise, alters LH pulsatility in exercising women. J. Appl. Physiol. 1998, 84, 37-46. [CrossRef] [PubMed]

52. Slater, J.; Brown, R.; McLay-Cooke, R.; Black, K. Low energy availability in exercising women: Historical perspectives and future directions. Sports Med. 2017, 47, 207-220. [CrossRef] [PubMed] 
53. Hilton, L.K.; Loucks, A.B. Low energy availability, not exercise stress, suppresses the diurnal rhythm of leptin in healthy young women. Am. J. Physiol. Endocrinol. Metab. 2000, 278, E43-E49. [CrossRef] [PubMed]

54. Hackney, A.C.; Fahrner, C.L.; Stupnicki, R. Reproductive hormonal responses to maximal exercise in endurance-trained men with low resting testosterone levels. Exp. Clin. Endocrinol. Diabetes 1997, 105, 291-295. [CrossRef] [PubMed]

55. Fahrenholtz, I.L.; Sjödin, A.; Benardot, D.; Tornberg, Å.B.; Skouby, S.; Faber, J.; Sundgot-Borgen, J.K.; Melin, A.K. Within-day energy deficiency and reproductive function in female endurance athletes. Scand. J. Med. Sci. Sports 2018, 28, 1139-1146. [CrossRef]

56. Torstveit, M.K.; Fahrenholtz, I.; Stenqvist, T.B.; Sylta, Ø.; Melin, A. Within-day energy deficiency and metabolic perturbation in male endurance athletes. Int. J. Sport Nutr. Exerc. Metab. 2018, 28, 419-427. [CrossRef]

57. Hawley, J.A.; Burke, L.M. Effect of meal frequency and timing on physical performance. Br. J. Nutr. 1997, 77 (Suppl. 1), S91-S103. [CrossRef]

58. Melin, A.; Tornberg, Å.B.; Skouby, S.; Møller, S.S.; Faber, J.; Sundgot-Borgen, J.; Sjödin, A. Low-energy density and high fiber intake are dietary concerns in female endurance athletes. Scand. J. Med. Sci. Sports 2016, 26, 1060-1071. [CrossRef]

59. Gordon, C.M.; Ackerman, K.E.; Berga, S.L.; Kaplan, J.R.; Mastorakos, G.; Misra, M.; Murad, M.H.; Santoro, N.F.; Warren, M.P. Functional hypothalamic amenorrhea: An endocrine society clinical practice guideline. J. Clin. Endocrinol. Metab. 2017, 102, 1413-1439. [CrossRef]

60. Enns, D.L.; Tiidus, P.M. The influence of estrogen on skeletal muscle: Sex matters. Sports Med. 2010, 40, 41-58. [CrossRef]

61. Elliott-Sale, K.J.; Tenforde, A.S.; Parziale, A.L.; Holtzman, B.; Ackerman, K.E. Endocrine effects of relative energy deficiency in sport. Int. J. Sport Nutr. Exerc. Metab. 2018, 28, 335-349. [CrossRef] [PubMed]

62. Tornberg, Å.B.; Melin, A.; Koivula, F.M.; Johansson, A.; Skouby, S.; Faber, J.; Sjödin, A. Reduced neuromuscular performance in amenorrheic elite endurance athletes. Med. Sci. Sports Exerc. 2017, 49, 2478-2485. [CrossRef] [PubMed]

63. Vanheest, J.L.; Rodgers, C.D.; Mahoney, C.E.; De Souza, M.J. Ovarian suppression impairs sport performance in junior elite female swimmers. Med. Sci. Sports Exerc. 2014, 46, 156-166. [CrossRef] [PubMed]

64. Harber, V.J.; Petersen, S.R.; Chilibeck, P.D. Thyroid hormone concentrations and muscle metabolism in amenorrheic and eumenorrheic athletes. Can. J. Appl. Physiol. 1998, 23, 293-306. [CrossRef]

65. Zinn, C.; Schofield, G.; Wall, C. Development of a psychometrically valid and reliable sports nutrition knowledge questionnaire. J. Sci. Med. Sport 2005, 8, 346-351. [CrossRef] 